\title{
Best practices and a working model for promoting inclusion of women in healthcare leadership
}

\author{
Alanna Dorsey*1, Rona Lee ${ }^{2}$, Wendy Zheng ${ }^{3}$, Magali Fassiotto ${ }^{4}$ \\ ${ }^{1}$ Stanford University, United States \\ ${ }^{2}$ Flourish All, United States \\ ${ }^{3}$ Carmenta Care, United States \\ ${ }^{4}$ Office of Faculty Development and Diversity, Stanford Medicine, United States
}

Received: December 14, 2021

DOI: $10.5430 /$ jha.v10n6p12
Accepted: January 17, 2022

Online Published: January 24, 2022

\begin{abstract}
There is a growing demand to increase the representation and empowerment of female leaders, and companies must implement effective policies to rise to the challenge. This article presents a potent new set of DEI (diversity, equity, and inclusion) protocols for healthcare administration to meet this challenge. The paper evaluates DEI practices and provides suggestions on advancing metrics such as recruitment, engagement and retention of women employees. We conducted a literature review and interviewed field experts to investigate best practices for shaping an inclusive healthcare leadership team. We identified four recurring themes, which are the key takeaways for successfully implementing any DEI initiative: 1. Garner support from the CEO and Board of Directors to establish the importance of the initiative throughout the company. 2. Engage employees directly; lead participants in designing diversity initiatives and encourage them to contribute their own ideas, rather than just going through the motions. 3. Involve the entire workforce, not just the top managers. As a definition of inclusion, everyone's perspective is essential for building a widespread work culture that exemplifies DEI principles. 4. Design DEI protocols that encompass life both in and out of the office, such as assisting women leaders with childcare needs. We then examine the most common DEI strategies: diversity training, employee resource groups, mentorship programs, and leadership development. Though these methods have their merits and shortcomings, expert input can mitigate the pitfalls. Lastly, we validate research-based interventions. According to the literature, healthcare has not adequately taken advantage of sponsorship opportunities, so we designed an executive-emerging leader sponsorship program. This protocol is supplemented with other interventions, such as interactive diversity training and ERG (employee resource group) playbooks, to foster the workspace crucial to the flourishing of program participants. Overall, we conducted secondary research on the best DEI protocols available, and augmented our findings with interviews we conducted. Therefore the findings we share are based on limited knowledge and do not represent the entire solution to diversity, equity and inclusion in healthcare leadership. Based on the best practices we are aware of, we present a multi-pronged approach to help healthcare administration shape a more equitable future for people of all backgrounds.
\end{abstract}

Key Words: Diversity, Women, Inclusion, DEI, Healthcare, Protocol

\section{INTRODUCTION}

The necessity of diversity in the workplace has been wellestablished. Companies with more female representation financially outperform those without. ${ }^{[1]}$ A Harvard Business Review article surveyed examples of how nonhomogenous teams make smarter decisions, and companies with more

*Correspondence: Alanna Dorsey; Email: alanna8@stanford.edu; Address: Stanford University, Stanford, United States. 
women are more likely to introduce innovative products. ${ }^{[2]}$ Across markets, studies found that companies with more diverse leadership report higher performance. ${ }^{[3]}$ However, it's not just about representation; healthcare organizations must be mindful of the environment they create. Across disciplines and demographics, employees who feel they have an equal, fair opportunity to advance report more career satisfaction, and are more likely to stay with their company. ${ }^{[4]}$ Healthcare systems need transformational leadership to meet the evolving needs of patients, and women are more likely than men to display these traits such as inspiring followers and leading by example. ${ }^{[5]}$ As the necessity for diversity and inclusiveness in the workplace has grown, so has the accumulation of DEI strategies. Like previous initiatives in digital health, ${ }^{[6]}$ we built a working model for achieving equity and inclusion in healthcare. We sought to research which protocols work and how they can be implemented effectively within healthcare leadership teams.

\section{MethodS}

For the literature review, we searched for articles regarding analysis of the efficacy of diversity programs, benefits of DEI, and statistics regarding DEI in healthcare. Content sources included academic journals, business magazines, and consulting firms. This search yielded 19 articles, 14 of which were used in the development of this study. We contacted DEI leaders at Stanford Healthcare for expert input. The interviewees included an Associate Dean for Faculty Development and Diversity in Stanford University's School of Medicine; an Inclusion, Diversity, and Equity leader at Stanford Healthcare, who is also the founder of Lean In Latinas; and the Diversity, Equity and Inclusion Program Manager at Stanford Children's Health. We conducted 1-2 interviews with each expert, asking about their challenges and successes in their DEI programs, as well as recommendations they have regarding the implementation of protocols.

\section{RESUlts}

\subsection{Best practices recommended by DEI experts}

With the expertise of leaders in DEI at one academic medical center, encompassing both school- and hospital-specific constituencies, we identified four overarching themes crucial to the success of diversity initiatives within healthcare.

\subsubsection{Executive sponsorship is key}

The literature emphasizes the importance of healthcare leaders in spearheading diversity efforts. CEOs must be committed to DEI, prioritizing it as a business movement rather than a role delegated to HR. As researchers in business leadership advise, CEOs must set clear, tangible goals for their company's inclusive environment, then hold managers acPublished by Sciedu Press countable for these milestones. ${ }^{[7]}$ With the support of CEOs, diversity becomes a business priority with real results.

All three DEI leaders agree: garnering support from senior leaders is pivotal for driving success. When executives, managers, and other senior leadership insist on the importance of DEI, employees are motivated to carve out time for DEIrelated activities like employee resource groups (ERGs). Not every company is in a similar situation, and corporations with less leadership investment may struggle to persuade employees to prioritize the initiatives. In such cases, organizations should introduce metrics to hold leaders accountable to DEI mission areas.

\subsubsection{Engage employees instead of using a top-down ap- proach}

Social scientists at Harvard found that many diversity programs fail due to their passive-rather than interactive-nature. ${ }^{[8]}$ Mandatory anti-discrimination training can compel people to rebel against policies or even reactivate bias. Allowing employees to opt-in to diversity training is more effective, as participants are more open-minded and willing to learn. However, the best option is directly involving managers and employees in diversity initiatives. Engagement in new programs empowers employees and makes them feel their work is meaningful, increasing investment in the final outcome.

Intentionality is crucial when engaging colleagues in the change process. In the academic healthcare space, for example, departments are often independently run, but must work together to make change. When working with this group, department chairs should be encouraged to strategize autonomously and to bring their own ideas to the table rather than passively absorbing directives from higher management.

\subsubsection{Include everyone in the picture, not just top manage- ment}

When implementing new DEI programs, open these to everyone. (However, in accordance with the previous tip, make sure DEI programs are framed as an opportunity, not a mandatory working group.) Assemble all stakeholders in employee resource groups. For healthcare, don't just get input from managers; include perspectives from nurses and physicians. A Deloitte report suggests not only training top managers and C-suite leaders; middle managers should also be trained in inclusion. ${ }^{[9]}$ One hospital that underwent a massive diversity program and saw impressive results advises other hospitals to train the entire staff. ${ }^{[10]}$ Though diversity leadership and other measures improved markedly, this hospital's staff could not achieve maximum impact because their budget constrained them to training only the management and staff of one nursing unit. Engaging the entire healthcare 
staff is crucial for building initiatives effective at all levels of the organization, and also boosts buy-in throughout the company.

\subsection{Inclusion initiatives must be all-encompassing, in and out of the office}

One challenge DEI officers face is employees perceiving DEI as an entity separate from their everyday work. As a solution, individuals need to understand how DEI is directly relevant to their jobs. Healthcare leaders can also incorporate DEI principles into new employee onboarding to set the precedent of fostering inclusion in all aspects of work. When organizing leadership training, for example, it is essential to integrate DEI into every skill taught. DEI is clearly involved in every aspect of working with others and leading a team. Therefore, when discussing a leadership topic like negotiation, consider how negotiation practices may be affected by different aspects of one's social identity.

On the topic of expanding diversity, inclusion must address all facets of employee life, not just the workplace. For example, an ERG at Stanford Medicine sponsored an event to address the impact of the pandemic on working women, such as childcare needs. Other organizations also studied how to best support employees: the ACHE evaluated survey results to assess the efficacy of diversity initiatives on job satisfaction among women. ${ }^{[1]}$ Some of the most successful initiatives target work-life balance, such as subsidized child care and senior care resources. Equity, inclusion, and diversity must be interwoven into the fabric of everyday life, in and out of the workplace.

\subsection{Case studies: benefits and pitfalls of current prac- tices}

Through case studies of Stanford University School of Medicine, Stanford Healthcare, and Children's Health, along with our own literature review, we present the strengths of current DEI protocols as well as challenges to mitigate.

\subsubsection{Employee resource groups (ERGs)}

Each of the DEI experts we spoke with was involved in organizing employee resource groups (also known as ERGs, affinity groups, or colleague resource groups), and advocated for their value. ERGs build community for marginalized groups within the workplace, providing a safe space for people to talk about their experiences with discrimination and microaggressions. However, it's important to note ERGs are not just networking groups; they support marginalized communities through career development, supporting local businesses, celebrating heritage months, and long term change initiatives. The ERGs in the case studies tackled issues such as rebuilding trust in the healthcare institution, implementing education initiatives regarding the COVID-19 pandemic, and expanding healthcare access. Therefore, ERGs support the community both in and out of the office.

These groups mobilize employees to raise awareness about company-wide issues and advocate for policies. For example, the LGBTQ+ group advocated for policies for trans employees and HR benefits specific to LGBTQ+ members of the workforce. These groups can also be learning opportunities for people who are not members of the group, by providing a safe space to learn about other cultures and people from different backgrounds. For example, the Latinx group explained the meaning of Cinco de Mayo to colleagues, and the LGBTQ+ group hosted a discussion about why pronouns are important. As mentioned in the previous section, employees should be allowed to opt-in so they are learning out of curiosity, instead of perfunctorily fulfilling requirements. Overall, employee resource groups can be fruitful opportunities to create momentum in the workplace, while educating employees from a casual and humble perspective.

ERGs are not without fault. Since these ERGs are often voluntary, not everyone has the time to attend and prioritize these groups. Some organizations may be challenged by trying to motivate employees to participate in these extra activities without compensation for their time. As a solution, get buy-in from managers and executives to fast-track DEI efforts. Another challenge is that ERGs lack standardization. As employee-run groups, outcomes could vary drastically within the same company. As a remedy, we suggest an outcome-driven approach, along with equipping ERG leaders with a playbook designed with industry-specific goals.

\subsubsection{Diversity training}

Training sessions have been the de facto DEI method for decades, but research findings are often unclear or contradictory. Diversity trainings have been shown to be ineffective or at best, short-lived in their impacts. ${ }^{[8]}$ However, unconscious bias education or conversations-a facet of diversity trainings-have been correlated with more equal gender representation in companies. ${ }^{[4]}$ Therefore, the data surrounding diversity trainings is mixed.

Regardless, there's a reason why diversity trainings stick around: they are a helpful tool to begin or sustain a conversation on topics employees may feel uncomfortable or uncertain about. However, employee growth should not stop there. One DEI training is not the final answer, but instead can serve as a means to raise awareness about bias and continue conversations in the workplace. 


\subsubsection{Mentorship programs/rotations}

Mentorship can be beneficial for underrepresented employees, who get an inside look into coveted positions and access to a wider resource network. Mentors also benefit, because sponsoring a marginalized employee increases their investment in their mentee's success, decreasing their implicit bias. ${ }^{[8]}$ However, to reap the full benefits of mentorship, mentors should follow guidelines. Mentors in senior leadership positions should serve as sponsors, advocating for mentees and finding opportunities for them. For example, a diversity in digital health report found that Black entrepreneurs not only need mentorship, they also need sponsors for funding or connections to investors. ${ }^{[12]}$ In the healthcare industry, mentors could find open positions for them or do job shadowing. An ACHE study found that one of the most effective ways to boost job satisfaction is by providing rotations to elevate women to senior-level executive roles. ${ }^{[11]}$ The biggest barrier for women climbing the corporate ladder is promotion to manager, ${ }^{[4]}$ so mentors should use their influence to help mentees overcome this hurdle. Furthermore, employees are more likely to feel they have fair access to opportunities when they have managerial support, such as managers who showcase their work and advocate for them regularly. Outcomes should be monitored, and both mentors and mentees should be allowed to discontinue their relationship at regular intervals.

\subsubsection{Leadership programs}

Leadership programs are a popular way to get more women and employees of color in the pipeline for executive leadership. While some companies utilize classic approaches such as videos, classes, and certifications, leaders can also consider newer methods such as individualized coaching to help early leaders identify their strengths and create authentic goals. Our DEI leaders utilize interactive programs to equip underrepresented faculty with leadership skills. In one instance, the School of Medicine developed a junior leadership bootcamp series, which introduces early-career faculty to an inter-departmental community. They gain leadership skills and advice from leaders facing similar challenges. This discussion amongst early-career employees instills a sense of leadership in every space people are in, no matter how young they are. Our DEI leaders also spoke about an inclusive leadership workshop that aims to raise awareness about inclusive work culture. Only by refining the definition of inclusive leadership into actionable goals can this be put into practice. Leadership programs are an excellent opportunity to combine education, goal-setting, and networking.

However, like mentorship, leadership programs need to be followed by actionable outcomes to lead to real change. With programs involving mentorship and leadership, it can be easy to fall into the trap of running the programs a few times and expecting success. However, these are only tools to fortify the pipeline and equip minority employees for roles that executives ultimately have to open up to them.

\section{Discussion}

DEI protocols enable all employees to celebrate their authenticity and thrive at work. Employees report greater career satisfaction when they feel they have equal access to opportunities as their colleagues. ${ }^{[4]}$ However, in the healthcare industry, female representation increased by only $0.3 \%$ a year since 2014, far behind other industries. ${ }^{[1]}$ A statistical analysis of gender equity in healthcare management found that women were less likely than men to be promoted to senior management positions, and a third of female managers reported gender discrimination. ${ }^{[13]}$

As a result of the paucity of widespread improvement in healthcare leadership, we investigated current DEI practices, then proposed novel protocols. In this study, we conducted a literature review and interviewed DEI experts to study the most effective ways to cultivate inclusive work environments within healthcare.

\subsection{DEI proposal for healthcare: built on the foundation of mentoring}

We propose a multi-faceted approach to make opportunities equally accessible to all employees, while ensuring that women are well-trained in leadership abilities. When researching the specific needs of healthcare organizations, the literature asserts "this field is not investing enough in mentoring and leadership development, and that this lack of attention is even more pronounced for women and minorities." [14]

Therefore, we propose a two-year sponsorship program in which employees are matched with a colleague one or two rungs above them in the corporate ladder. Training will encompass not only sponsees but also sponsors, as many mentors have not learned how to be a sponsor. This mentorship program will be supplemented by programs that worked well in our case studies, providing a well-rounded, research-backed approach to hiring and retaining diverse talent. Similar leadership development and mentorship protocols for marginalized communities, such as ACHE's Thomas C. Dolan Executive Diversity Program, have proven beneficial for racial and ethnic minorities in healthcare, ${ }^{[15]}$ suggesting a positive outcome of our program for women.

\subsection{Pre-assessment}

At the start of the DEI initiative, the HR manager or DEI consultant must evaluate how well the healthcare organization meets DEI benchmarks. There are many DEI checklists avail- 
able, such as the AHA's diversity and cultural proficiency assessment for healthcare organizations. ${ }^{[16]}$ The officer should also evaluate how well faculty members are trained in cultural competency. After assessing where the organization currently stands, leaders can tailor the rest of this protocol to their specific needs. After pre-assessment and goal-setting, the officer coordinating the program is ready to approach executives about the protocol.

\section{Step 1: Get buy-in from execs}

Before implementing any new DEI programs, get the CEOs and the Board of Directors involved. If it is not already clear, let the executives know that DEI is a cultural change that takes commitment and patience, rather than being a one-off project. After securing the support and investment of the C-suite employees, invite them to take part in the mentorship program. Showing the rest of the company how involved the leadership is will encourage other employees to prioritize DEI efforts and take part in these programs.

\section{Step 2: Implement programs}

1. Executive-emerging leaders sponsorship program: while ERGs and diversity training have been widely practiced in healthcare, sponsorship programs have been less popular. However, sponsorship programs provide mentees with networking opportunities and mentors with a more inclusive mindset as they work more closely with people from diverse backgrounds. We propose a sponsorship program that pairs promising early employees with higher-ups, such as a manager paired with a director, or a director with a $\mathrm{C}$-suite executive. Mentees receive cultural competency training with physician speakers to gain hands-on experience while also earning medical education credits. This program would last for two or more years, with pairs meeting up every other month to check in and discuss strategies for promotion. To build credibility in the program, employees complete a short application and secure a recommendation before receiving a spot in the program. However, these sponsorship programs don't end with mentorship: mentees who complete the program will be put on the short list for available promotions, with their sponsors advocating for them. Mentees will also do inclusive leadership training (such as the six traits of inclusive leadership training, see below) funded through the learning and development department within HR. At the conclusion of the program, mentees will have networked with higher-ups, gained insider's advice in climbing the corporate ladder, and trained in building an inclusive workplace, putting them in the prime position for promotion.

(1) How the program will identify and recruit candidates: we propose identifying 30-50 participants from marginalized backgrounds (including but not limited to gender). Candidates (both mentors and mentees) will be evaluated based on their seniority, work experience, competence, and career aspirations. Personal factors to be considered include goals, adversity overcome, and past public service/mentoring experience.

(2) How the program will engage with participants: mentees will complete monthly DEI training workshops either through virtual video conferencing or in person. Part of this training will be based on Deloitte's article "The six signature traits of inclusive leadership". [17] This comprehensive guide instructs leaders to be active, curious listeners who try to understand where people are coming from and are aware of their own faults. DEI workshops should incorporate these six traits but also go above and beyond, covering topics such as culturally-specific issues within healthcare.

(3) At the conclusion of the program, participants will complete a capstone project to apply their learning and create tangible change in their workplace. Mentees will receive a DEI expertise certificate upon completion of the course, thus qualifying them for higher-up positions. Additionally, participants receive mentorship by the leaders of the workshops and their personal mentor during the course of the program.

2. ERG playbook and resource database: through Stanford Healthcare case studies, we noticed ERGs provide the unique opportunity for colleagues to learn from each other and commune in a safe space. For companies who have yet to implement ERGs, we propose providing a playbook to navigate setting up and sustaining a group. Pre-existing groups with a preferred flow should also be supported in their endeavors. We suggest providing a database or website of resources these ERGs can use for locating funding opportunities for community activities, guidelines to setting up task forces, and any other useful materials.

3. Interactive diversity training: as the bread and butter of DEI, diversity training is helpful but in need of revamping. Programs should directly engage employees (see "Best Practices Recommended by DEI Experts"). We propose interactive diversity programs for employees to experience the perspective of co-workers from other backgrounds. For example, teams could host employee open-mic nights, spotlights, or any kind of open forum that builds a supportive community. The focus of this program is to build empathy and active listening, lending to a more inclusive atmosphere.

\section{Step 3: Gather and analyze metrics}

One crucial aspect of successful protocols is establishing realistic expectations and metrics to analyze program efficacy. All our proposed programs will run for at least two 
years, after which we expect to see higher satisfaction and more diverse representation in staff. To measure this, the program coordinators will distribute pre- and post-surveys to employees. For the sponsorship program, coordinators will track how many were promoted during the program. Anonymous surveys will ask participants about the helpfulness of the trainings and how long they intend to stay with the company. Employees who participated in the ERGs and interactive diversity training will be asked how comfortable they feel at work discussing issues with being marginalized, and how comfortable they feel working with people from other backgrounds. Program coordinators will track if participants report a safer and more open work environment as a result of our interventions. Overall, data analysis will focus on measuring the promotion, retention, satisfaction, and recruitment of diverse candidates during the DEI protocol.

\section{Step 4: Host mentorship summit}

To conclude the program, we propose hosting a conference for participants. This summit can take place at various healthcare events, such as the ACHE Annual Conference. The reception will celebrate participants and provide networking opportunities to meet other healthcare professionals. With the partnership of a university, graduate students can also attend as part of their training in the field.

\section{Budget and ROI}

The cost of the program varies, based on length of engagement. For example, as part of the monthly DEI trainings for the sponsorship program, the budget could include $\$ 10 \mathrm{~K}$ for a one-day training by faculty development officers and healthcare experts, teaching skills such as inclusive leadership styles and managing culturally responsive clinical care. To measure ROI of the protocol, review the aforementioned post-protocol metrics.

\subsection{Limitations and future research}

One strength of this study is that we conducted in-depth interviews with experts at a leading healthcare organization. With their extensive combined experience within the field, all experts gave detailed advice and critical analyses of prior and ongoing programs. Additionally, some of these experts work in different branches of the institution: for example, one interviewee works in the office of faculty development and diversity, while another works in the children's hospital, and yet another works in the adult hospital. Consequently, the interviewees provided different perspectives based on their sector of healthcare administration.

However, one limitation is that all the selected experts work at the same institution, confining their experiences to a similar population and environment. Since the interviewees worked within the same ecosystem, our gathered responses did not yield as much diversity of thought as would be necessary for an entirely inclusive DEI recommendation. All our experts worked at Stanford Healthcare, a health system with a teaching hospital within a prominent academic institution. While their experiences and insight are valuable teaching opportunities, they are not necessarily representative of all health systems. Future research should be supplemented with interviews from objective DEI consultants outside of this ecosystem, such as those working with public hospitals or non-profit healthcare services unaffiliated with the Stanford institution. Researchers should consider interviewing experts from healthcare organizations throughout the state or nation, and comparing the advice to yield overarching themes and community-specific guidelines.

Another strength presented by our literature review is that we focused on gender and racially marginalized populations within healthcare management. This focus allowed us to take a deep dive into the unique problems faced by these populations. Further DEI research needs to examine issues for all genders, such as paid paternity leave and gender-neutral bathrooms. Another limitation is that gender and race are not the only marginalizing factors in the workplace. Future research should study how DEI protocols impact LGBTQ+ communities, those with disabilities, and other marginalized groups. The future of equity in healthcare rests in researchers recognizing the value of studying all groups, especially those who are understudied.

\subsection{Recommendations for healthcare leaders}

Through a literature review, interviews with experts, and case studies, we identified four keys to implementing protocols:

(1) Secure executive sponsorship; garnering CEO support helps ensure diversity becomes a priority.

(2) Directly engage employees; involve them in the process of changemaking instead of just telling them what to do.

(3) Invite all levels of employees to take part in programs, not just top managers.

(4) DEI initiatives must encompass all aspects of employee life.

\section{Blueprint of our proposal:}

(1) Secure support of C-suite executives and the Board of Directors.

(2) Implement the executive-emerging leaders sponsorship program, ERG resource list and database, and interactive diversity training.

(3) Gather metrics to analyze employee satisfaction and retention.

(4) Host a mentorship summit for sponsorship program par- 
ticipants to reflect on their experiences and celebrate their accomplishments at the conclusion of the two-year program.

However, healthcare leaders should be cautioned that this proposal alone is not a guarantee of perfect and enduring inclusivity in their workplace. Leaders engaging in this protocol must practice effective management strategies to support their employees and tailor the program to the specific needs of the institution. Leaders must also engage in careful planning and execution of this program to maximize success. Lastly, our expert recommendations and proposed strategy are merely a means to facilitate DEI efforts in healthcare; it is up to the leadership team to continue these conversations and ensure that advocacy and inclusive workspaces outlast the two-year program.

\section{Conclusions}

Given the benefits and necessity of inclusion, there is no better time to develop an equitable workplace. With our proposed interventions, we foresee healthcare organizations equipping minority leaders with the skills needed for promotion and fostering an inclusive work environment, paving the way for a more equitable future.

\section{ACKNOWLEDGEMENTS}

We thank Anna Dapelo-Garcia and Leslie Truong for their insights. We also thank the team members of Flourish All for their support and guidance.

\section{CONFlicts OF InTEREST Disclosure}

The authors declare they have no conflicts of interest.

\section{REFERENCES}

[1] Dixon-Fyle S, Dolan K, Hunt V, et al. Diversity wins: How inclusion matters [Internet]. McKinsey \& Company. 2020 [cited 2021 Dec 14]. Available from: https: //www.mckinsey.com/featured-insights/diversity-and -inclusion/diversity-wins-how-inclusion-matters

[2] Rock D, Grant H. Why Diverse Teams Are Smarter. Harvard Business Review. 2016; 4(4): 2-5.

[3] Hewlett SA, Marshall M, Sherbin L. How Diversity Can Drive Innovation. Harvard Business Review. 2013; 30.

[4] Huang J, Krivkovich A, Starikova I, et al. Women in the Workplace 2019. McKinsey \& Company. 2019; 1-10.

[5] McDonagh KJ, Bobrowski P, Hoss MA, et al. The leadership gap: Ensuring effective healthcare leadership requires inclusion of women at the top. Open Journal of Leadership. 2014; 3(2): 20-9. https://doi.org/10.4236/oj1.2014.32003

[6] Simmons D, Kaganoff S, Drasser K. Building toward equity: A Working Model for Digital Health: Rock health [Internet]. Rock Health. 2021 [cited 2021Dec14]. Available from: https://rockhealth.com/insights/building-towar d-equity-a-working-model-for-digital-health/

[7] Bersin J, Enderes K. Elevating Equity: The Real Story of Diversity and Inclusion. Josh Bersin Academy. 2021; 1-52.

[8] Dobbin F, Kalev A. Why Diversity Programs Fail. Harvard Business Review. 2016; 14.

[9] Bourke J, Dillon B. The diversity and inclusion revolution: Eight powerful truths. Deloitte Review. 2018; 22: 82-95.

[10] Dreachslin JL, Weech-Maldonado R, Gail J, et al. Blueprint for Sustainable Change in Diversity Management and cultural competence.
Journal of Healthcare Management. 2017; 62(3): 171-83. PMid: 28471853. https://doi.org/10.1097/JHM-D-15-00029

[11] Athey LA. Addressing gender equity in healthcare organizations [Internet]. American College of Healthcare Executives. Foundation of the American College of Healthcare Executives; 2019 [cited 2021 Dec 14]. Available from: https ://www . ache.org/-/media/ache/learning-cen ter/research/2019-ceo-circle-white-paper.pdf

[12] Horn IB, Parikh P, Zweg M. Diversity in Digital Health. Rock Health. $2020 ; 1-58$

[13] LaPierre TA, Zimmerman MK. Career Advancement and Gender Equity in healthcare management. Gender in Management: An International Journal. 2012; 27(2): 100-18. https://doi .org/10.110 $8 / 17542411211214158$

[14] Lantz PM. Gender and leadership in healthcare administration: 21st Century progress and challenges. Journal of Healthcare Management 2008; 53(5): 291-301. PMid: 18856135. https://doi.org/10.1 097/00115514-200809000-00004

[15] Zambrano RH. The Value and Imperative of Diversity Leadership Development and Mentoring in Healthcare. Journal of Healthcare Management. 2019; 64(6): 356-8. PMid: 31725561. https : //doi .org/10.1097/JHM-D-19-00209

[16] American Hospital Association. Strategies of leadership: Does your hospital reflect the community it serves. 2004. Available from: https://www.aha.org/system/files/2018-02/Dive rsityTool.pdf

[17] Dillon B, Bourke J. The six signature traits of inclusive leadership. Deloitte University Press; 2016. 1-26 p. 